\title{
Growth Regimes of Poly(perfluorodecyl acrylate) Thin Films by Initiated Chemical Vapor Deposition
}

\author{
Alberto Perrotta, ${ }^{\circledR}$ Paul Christian, Andrew O. F. Jones, ${ }^{\circledR}$ Fabian Muralter, and Anna Maria Coclite*(i) \\ Institute of Solid State Physics, NAWI Graz, Graz University of Technology, Petersgasse 16, 8010 Graz, Austria
}

Supporting Information

ABSTRACT: Control over thin film growth (e.g., crystallographic orientation and morphology) is of high technological interest as it affects several physicochemical material properties, such as chemical affinity, mechanical stability, and surface morphology. The effect of process parameters on the molecular organization of perfluorinated polymers deposited via initiated chemical vapor deposition (iCVD) has been previously reported. We showed that the tendency of poly $(1 \mathrm{H}, 1 \mathrm{H}, 2 \mathrm{H}, 2 \mathrm{H}$-perfluorodecyl acrylate) (pPFDA) to organize in an ordered lamellar structure is a function of the filament and substrate temperatures adopted during the iCVD process. In this contribution, a more thorough investigation of the effect of such parameters is presented, using synchrotron radiation grazing incidence and specular X-ray diffraction (GIXD and XRD) and atomic force microscopy (AFM). The parameters influencing the amorphization, mosaicity, and preferential orientation are addressed. Different growth regimes were witnessed, characterized by a different surface structuring and by the presence of particular crystallographic textures. The combination of morphological and crystallographic analyses allowed the identification of pPFDA growth possibilities between island or columnar growth.

\section{INTRODUCTION}

Low-surface-energy compounds are a class of materials showing peculiar physicochemical properties, such as hydrophobicity and oleophobicity, which make them appealing for a wide variety of applications, from bioinspired and biocompatible materials ${ }^{1-3}$ to anticorrosion ${ }^{4,5}$ and antifouling coatings, ${ }^{6}$ self-cleaning surfaces, ${ }^{7}$ and permeation membranes. ${ }^{8}$

Fluorine-containing polymers are a subclass of low-surfaceenergy materials which have gained particular attention in materials science due to their intrinsic hydrophobicity as well as peculiar morphology and surface structure. ${ }^{9-12}$ Perfluorinated chains in (co)polymers often result in strongly apolar surfaces with reduced wettability for both water and/or organic molecules, reduced adhesion, and excellent antisticking properties due to the low surface energy and strong chemical inertness. ${ }^{13}$ Processing of fluorinated thin films can be achieved with a variety of techniques, ${ }^{14}$ and among these, vapor-phase techniques have shown several advantages, such as the solventless processing and control over the chemical composition and structural properties of the thin films by acting on easily adjustable process parameters. ${ }^{12,15}$ Furthermore, it has been shown that the growth of fluorinated polymers on nanostructured substrates, together with the substrate-independent thin film technologies, allowed for unique surface structures, leading to properties like superhydrophobicity and oleophobicity. ${ }^{16}$

Among the thin film technologies, initiated chemical vapor deposition (iCVD) has been increasingly employed for the deposition of perfluorinated polymers. ${ }^{17,18}$ In the iCVD technique, a radical initiator and monomer(s) are fed into a vacuum chamber from the vapor phase. The thermal decomposition of the radical initiator (generally a peroxide) occurs in the gas phase and is driven by a filament heated to 200-350 ${ }^{\circ} \mathrm{C}$, suspended over a temperature-controlled substrate. Temperatures in this range have been demonstrated to affect only the labile peroxide bond of the initiator molecules while leaving the monomer structure intact. ${ }^{19}$ The initiator radicals then attack the unsaturated bonds of the monomers adsorbed on the substrate (due to their generally low vapor pressure). Subsequent chain growth and polymerization reactions follow the path of a classical radical polymerization, although occurring solely on the substrate surface. The advantages of $\mathrm{iCVD}$ compared to other vaporbased deposition techniques (e.g., PE-CVD or physical vapor deposition) are the ability to finely control the possible reaction pathways and, most importantly, to fully retain the characteristic functional groups of the monomer.

For the deposition of perfluorinated polymers via iCVD, $1 \mathrm{H}, 1 \mathrm{H}, 2 \mathrm{H}, 2 \mathrm{H}$-perfluorodecyl acrylate (PFDA, Figure 1a) has been largely used. ${ }^{17,20-23}$ Its fast polymerization of the unsaturated acrylate group as well as excellent thermal and chemical stability ${ }^{24}$ makes it suitable for the synthesis of different polymers and copolymers and has already been applied for separation membranes, ${ }^{22}$ proton conductive membranes, ${ }^{21,25}$ and corrosion protection. ${ }^{5}$

Received: April 27, 2018

Revised: June 11, 2018

Published: July 20, 2018 
a)

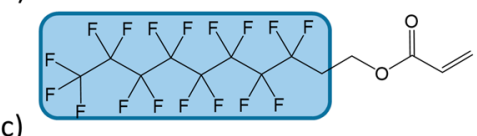

C)
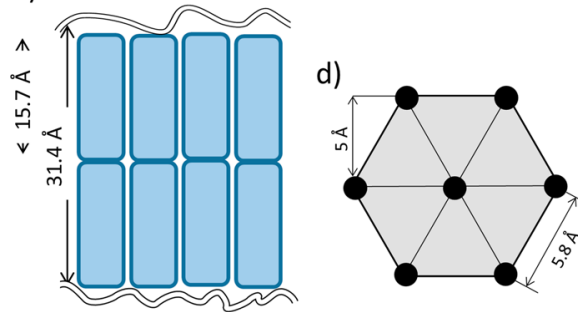

b)

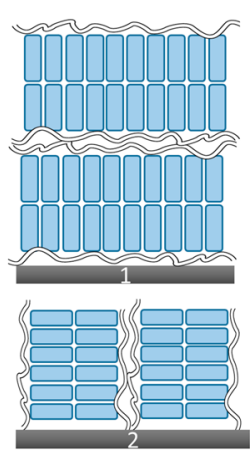

Figure 1. (a) Chemical structure of the PFDA monomer, with the fluorinated group highlighted (b) two characteristic preferential orientations of the pPFDA bilayer, with the lamellae either parallel (1) or perpendicular (2) to the substrate surface. (c) Smectic B structure of pPFDA, forming a lamellar structure with a periodicity of $31.4 \AA$. (d) Hexagonal in-plane structure of the crystalline phase of pPFDA (top view).

Furthermore, next to the chemical characteristics, polyPFDA (pPFDA) has been demonstrated to possess peculiar crystallographic and morphological properties. It is well-known that the number of $\mathrm{CF}_{2}$ repeating units in the pendant groups determines the hydrophobic/oleophobic properties of the resulting polymer. ${ }^{24,26}$ These properties arise from the phase separation and self-organization of the perfluorinated chains as polymer segments (i.e., the pendent groups and the polymer backbone) are chemically incompatible. The polymer system arranges all segments in phases with specific order and distance relationships. In the literature, it has been shown that perfluorinated chains longer than seven $\mathrm{CF}_{x}$ units form aggregates in an ordered lamellar structure called a smectic

phase. $^{24}$ For pPFDA polymers, a smectic B phase was identified (Figure 1c,d), in which perfluorinated side groups are packed in bilayers. In the literature, different values for the bilayer periodicity have been reported as a function of the deposition method and experimental condition adopted. $^{10,24,26,27}$ Generally, polymers synthesized or deposited using a solvent (e.g., spin-coated, casted) showed higher periodicity, in between 34 and $33.2 \AA^{24,26,27}$ Instead, we previously measured shorter periodicity $(31.8 \AA)$ in other iCVD pPFDA layers, suggesting that the absence of solvents affects the chain organization and crystal formation. Next to the bilayer, the pendant groups also assume a hexagonal packing within the lamella, with a lattice parameter of $5.8 \AA$ (Figure 1d).

In a previous contribution, we studied the effect of iCVD process parameters on the degree of crystallinity and crystallographic orientation of pPFDA. ${ }^{10}$ Parameters such as the initiator-to-monomer flow ratio and the filament and substrate temperatures have been shown to strongly affect the crystallographic order and the morphology of the polymeric thin films. By tuning the process parameters, two different preferred orientations of the perfluorinated lamellae were identified, being either parallel or perpendicular to the substrate surface (Figures 1c, 1, and 2, respectively). Different wettability and morphology were observed, depending on the preferred orientation, in this way obtaining polymers with controllable physicochemical properties.

The aim of this study is to further investigate the effect of the iCVD parameters on the crystalline arrangement of pPFDA polymers, shedding light on the possible growth modes of pPFDA and additional possibilities to control the properties of the polymer. In particular, more detailed analyses were carried out on the effect of both substrate and filament temperature on
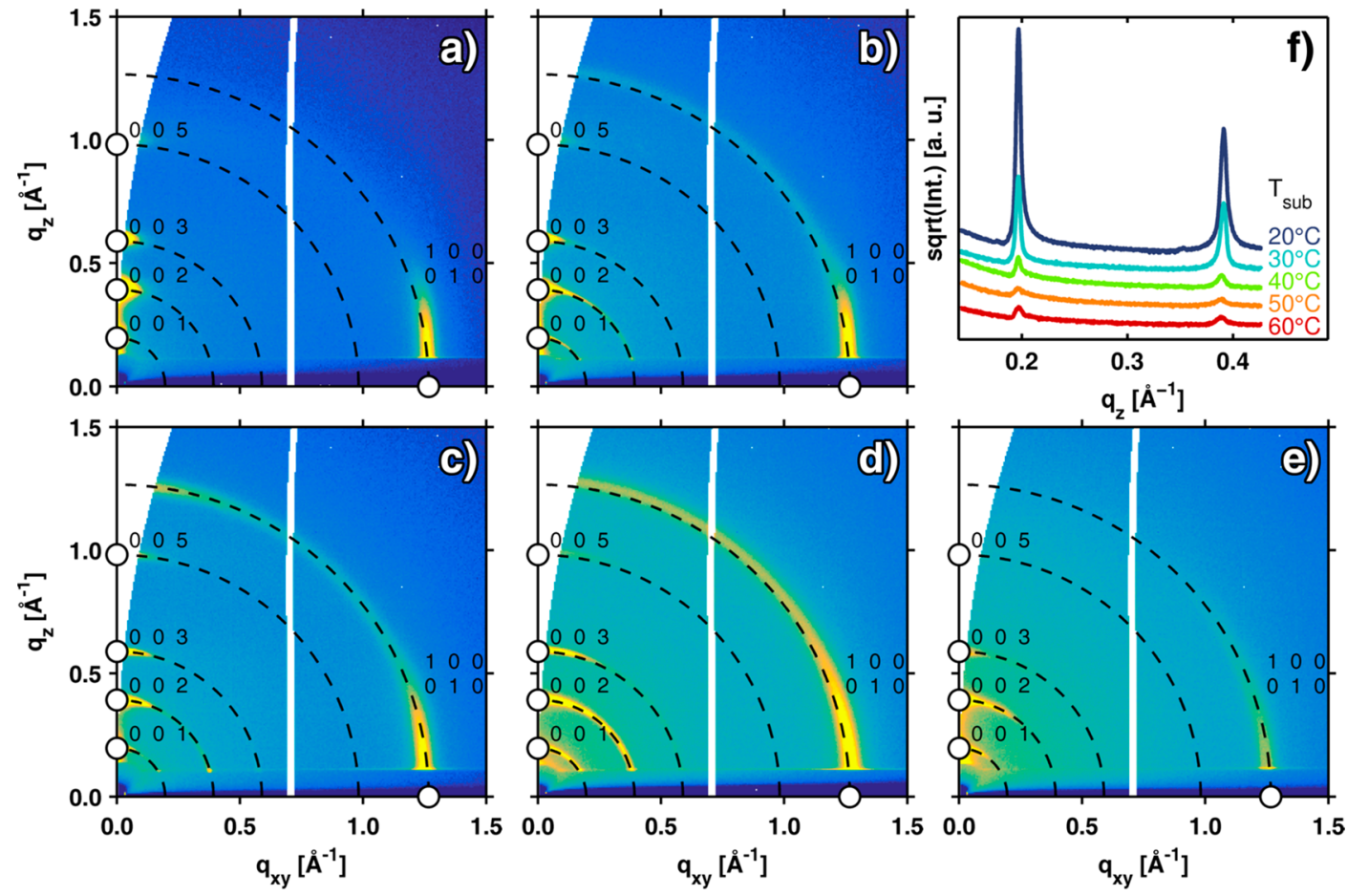

Figure 2. GIXD data for thin pPFDA films deposited by iCVD at substrate temperatures of (a) 20, (b) 30, (c) 40, (d) 50, and (e) $60{ }^{\circ} \mathrm{C}$. The filament temperature was $280^{\circ} \mathrm{C}$ for all the depositions. (f) Specular XRD patterns of the samples. Data have been shifted vertically for the sake of clarity. 
the crystallographic orientation and morphology of the perfluorinated polymers. The study expanded what has been discussed in the literature and allows further insight into the iCVD technique and into the effect of its process parameters on material properties.

\section{EXPERIMENTAL SECTION}

Polymer coatings of $1 \mathrm{H}, 1 \mathrm{H}, 2 \mathrm{H}, 2 \mathrm{H}$-perfluorodecyl acrylate (PFDA, $97 \%$, Aldrich, Germany) were deposited in a custom-built initiated chemical vapor deposition (iCVD) chamber, using tert-butyl peroxide (TBPO, 98\%, Aldrich, Germany) as initiator. The cylindrical chamber (height $5.5 \mathrm{~cm}$, diameter $36 \mathrm{~cm}$ ) is pumped by a Duo 65 rotary vane pump (Pfeiffer Vacuum, Germany), and the process pressure is regulated by a throttle valve (MKS Instruments, USA). A removable quartz glass lid allows in situ thickness control via laser interferometry ( $\mathrm{He}-\mathrm{Ne}$ laser with $\lambda=633 \mathrm{~nm}$, Thorlabs, USA). The chamber houses a resistively heated filament array of 12 parallel nickelchromium wires (Goodfellow, UK), which are mounted with a clearance of $2.5 \mathrm{~cm}$ above the bottom of the reactor, used as sample holder. The filament temperature is monitored by a spot-welded type $\mathrm{K}$ thermocouple (Omega Engineering, USA). The substrate temperature is regulated by an Accel $500 \mathrm{LC}$ heater/chiller (Thermo Fisher Scientific, USA) to $\pm 2{ }^{\circ} \mathrm{C}$, as monitored by a type $\mathrm{K}$ thermocouple. The PFDA monomer is stored in a glass jar, kept at $80^{\circ} \mathrm{C}$, and flown into the reactor through a heated line (held at $90{ }^{\circ} \mathrm{C}$ ). The radical initiator TBPO is kept at room temperature. Nitrogen is used as carrier gas. The PFDA flow rate was manually set by a needle valve (Swagelok, USA). The initiator and carrier gas flow rates were set through mass flow controllers (MKS Instruments, USA). In Table 1, the process parameters used in this work are reported.

Table 1. Experimental Conditions Used for the Growth of PPFDA Thin Films by iCVD

\begin{tabular}{|c|c|c|c|c|c|c|}
\hline & $\begin{array}{l}\text { PFDA } \\
{[\mathrm{sccm}]}\end{array}$ & $\begin{array}{l}\text { TBPO } \\
{[\mathrm{sccm}]}\end{array}$ & $\underset{[\mathrm{sccm}]}{\mathrm{N}_{2}}$ & $\begin{array}{c}T_{\text {filament }} \\
{\left[{ }^{\circ} \mathrm{C}\right]}\end{array}$ & $T_{\text {substrate }}\left[{ }^{\circ} \mathrm{C}\right]$ & $\begin{array}{l}\text { press. } \\
{[\mathrm{mTorr}]}\end{array}$ \\
\hline $\begin{array}{r}T_{\text {substrate }} \\
\text { series }\end{array}$ & 0.20 & 0.6 & 4.0 & 285 & $20-60$ & 800 \\
\hline \multirow{2}{*}{$\begin{array}{r}T_{\text {fliment }} \\
\text { series }\end{array}$} & 0.26 & 0.6 & 4.0 & $240-360$ & 30 & 800 \\
\hline & 0.26 & 0.6 & 4.0 & $240-360$ & 50 & 800 \\
\hline
\end{tabular}

All samples were deposited on single-side polished crystalline silicon wafers (Siegert Wafer) with a $1.5-2 \mathrm{~nm}$ thick native oxide layer on top. Coatings with an average thickness of $220 \pm 30 \mathrm{~nm}$ were deposited. For the layers deposited at different substrate temperatures, the amount of monomer adsorbed on the surface, generally reported as the ratio between the monomer partial pressure $\left(P_{\mathrm{M}}\right)$ and its saturation pressure $\left(P_{\text {sat }}\right)$, was not set to a specific value (see Table $S 1)$. For the layers deposited at different filament temperature, the amount of adsorbed monomer was fixed to a specific value for each of the substrate temperatures investigated. The specific effect of the monomer adsorption on the crystallinity and texture of PPFDA thin films was not investigated due to the inconclusive correlation of the $P_{\mathrm{M}} / P_{\text {sat }}$ parameter with the crystal growth, inferred by both literature $^{5,10}$ and the present results. More details are given in the Supporting Information.

The thickness of the thin films was measured ex situ by variable angle spectroscopic ellipsometry (M-2000S, J.A. Woollam) at three different angles $\left(65^{\circ}, 70^{\circ}\right.$, and $\left.75^{\circ}\right)$ in the wavelength range $370-$ $1000 \mathrm{~nm}$. The optical model chosen consists of three layers: the c-Si as substrate, native $\mathrm{SiO}_{2}$ (with thickness in the range $1.5-2 \mathrm{~nm}$ ), and the pPFDA thin film. The optical properties of the latter were modeled by a Cauchy function, with an Urbach tail accounting for absorption at lower wavelength.

The crystalline sample properties were investigated with a PANalytical Empyrean X-ray diffractometer, equipped with a copper sealed tube (wavelength $\lambda=0.154 \mathrm{~nm}$ ), a Göxbbel mirror, various slits, and a PIXcel ${ }^{3 \mathrm{D}}$ solid-state detector operated in receiving slit mode. Detailed scans were taken in the scanning line mode, which detects also some off-specular intensity ( spread $\left.\Delta \omega= \pm 1.65^{\circ}\right)$. The angular scans $(\theta / \theta)$ are represented in the scattering vector $\left(q_{z}\right)$ notation, whereby $q_{z}=4 \pi \sin (\theta) / \lambda$. Such a representation allows a direct comparison of measurements taken at other wavelengths. The index $z$ denotes that only net-planes parallel to the substrate surface are evaluated (specular scans).

Atomic force micrographs were taken in noncontact mode on a Nanosurf easyScan 2, equipped with a PPP-NCLR-10 cantilever (NanoWorld AG, Switzerland). The data are corrected for artifacts with the freely available software package Gwyddion. ${ }^{28}$

Grazing incidence X-ray diffraction (GIXD) was performed to investigate the in-plane orientation of the crystallites. The measurements were conducted at the Elettra XRD1-beamline in Trieste, Italy. ${ }^{29}$ The incident angle $\alpha$ was set between $1.2^{\circ}$ and $2^{\circ}$ for optimal signal intensity, and the wavelength $\lambda$ of the primary beam was either 1 or $1.4 \AA$. Diffracted intensities were collected on a Pilatus $2 \mathrm{M}$ detector, and all data have been recalculated to (wavelengthindependent) reciprocal space maps using the in-house developed software package GidVis. In such maps, intensities are plotted in a pseudocolor representation as a function of the specular $\left(q_{z}\right)$ and the in-plane component $\left(q_{x y}\right)$ of the scattering vector. For sake of clarity and comparability, all intensity data were plotted in square root representation and are represented on same color scales.

\section{RESULTS AND DISCUSSION}

The iCVD technique has been used for the synthesis of pPFDA thin films following the procedure previously reported. $^{21,30}$ Full retention of the monomer structure and conversion of the unsaturated bonds were witnessed by Fourier transform infrared spectroscopy (FT-IR). The results are in agreement with successful polymerization also reported in previous studies. $^{30,31}$ X-ray photoelectron spectroscopy (XPS) was also used as an additional tool to verify the retention of the PFDA functional groups. Both FT-IR and XPS spectra are reported in the Supporting Information. In order to investigate the structural and morphological changes in the pPFDA thin films as a function of the experimental parameters, the effects of the substrate and filament temperature were studied. In the literature, the ratio of the monomer to the initiator flow (I/M) was shown to greatly affect the structural and morphological properties of the pPFDA films. ${ }^{10}$ This effect was not further investigated in the current study, and for this reason, the I/M was kept in the range $2-2.5$ where high crystallinity can be achieved.

Substrate Temperature Effect. pPFDA layers were deposited varying the substrate temperature in the range 20-60 ${ }^{\circ} \mathrm{C}$ (Table 1). Previous investigations showed that lower substrate temperatures would result in increased crystallinity when measured in a specular XRD scan (that is, measuring crystallographic planes parallel to the substrate). Increasing the substrate temperature would dramatically reduce the crystallinity, resulting in thin films with smoother surfaces as measured with AFM. ${ }^{10}$ In order to gain further insight into the crystal growth behavior, GIXD images and specular scans were acquired, in this way gathering information on both the in-plane and out-of-plane crystal orientations. In Figure 2, both GIXD and specular XRD data are presented for pPFDA films prepared at different substrate temperatures.

All the peaks reported in Figure 2 correspond to aggregates in the ordered smectic B phase (Figure 1). From the specular scans reported in Figure $2 \mathrm{f}$, the first-order $\left(q_{z}=0.20 \AA^{-1}\right)$ and second-order $\left(0.40 \AA^{-1}\right)$ Bragg peaks of the bilayer structure can be observed. These peaks are characteristic for (fluoroalkyl acrylate) polymers (Figure $1 \mathrm{~b}$ ) and correspond to spacings of 

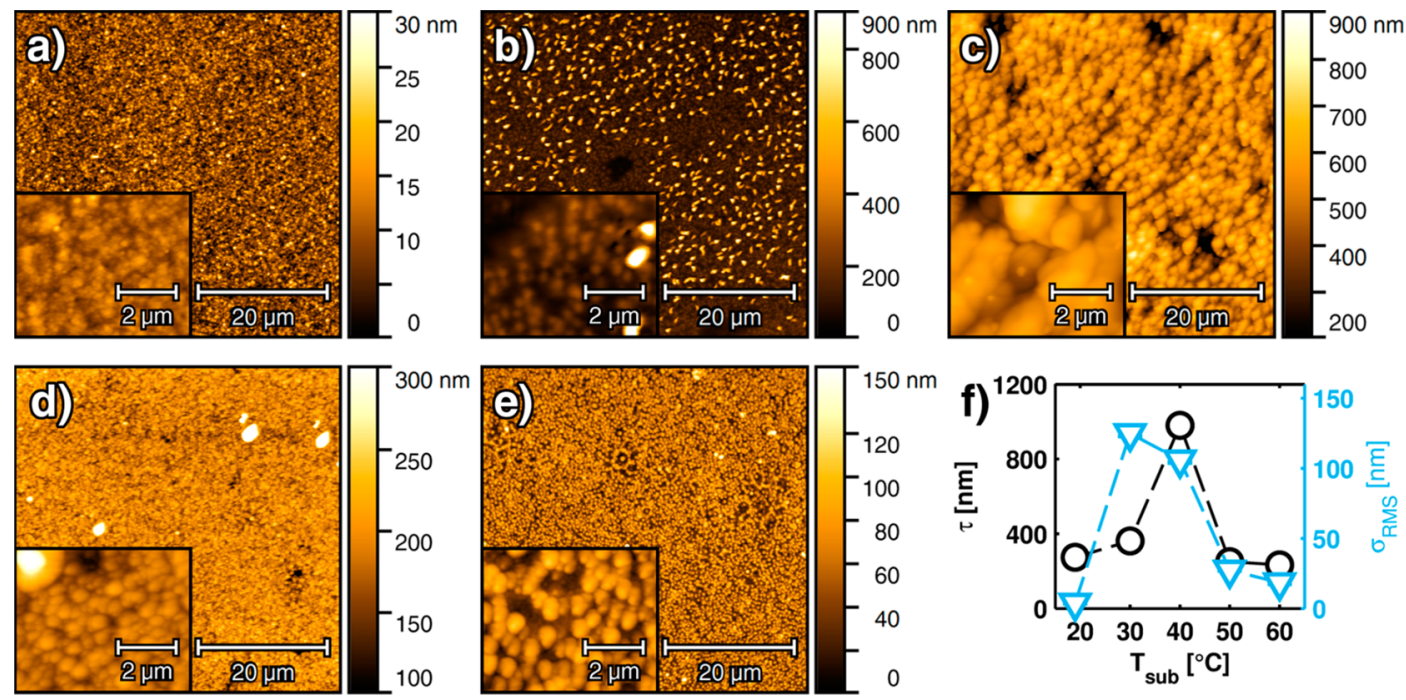

Figure 3. Topographical AFM images for iCVD pPFDA thin films deposited at substrate temperatures of (a) 20, (b) 30, (c) 40, (d) 50, and (e) 60 ${ }^{\circ} \mathrm{C}$. High-resolution detailed views are provided in the insets. (f) Surface roughness $\sigma_{\text {RMS }}$ and the autocorrelation length $\tau$ as determined from analysis of the radial power spectral density function (rPSDF) of the data. Please note that the inset depicted in (b) uses a different color range for the sake of visibility.

31.4 Å (bilayer) and $15.7 \AA$ (single layer lamella). This bilayer distance is slightly lower than that of pPFDA synthesized by conventional radical polymerization, for which values between 33 and $34 \AA$ are reported. ${ }^{24,26,27}$ The absence of solvents and the possible presence of residual stress in the films could account for the differences in packing. A detailed specular scan depicting second- and third-order Bragg reflections is provided in the Supporting Information (Figure S3). The trend in crystallinity as a function of the substrate temperature is in agreement with the investigation reported in the literature (Figure 2f) for specular scans. A higher degree of crystallinity is found at lower temperatures (e.g., at $20^{\circ} \mathrm{C}$ ), while an increase in the substrate temperature leads to a decrease in crystallinity. To assign the decrease in crystallinity either to an overall amorphization of the layer or to a reorientation of the crystal structure, GIXD measurements were performed on the same samples (Figure 2a-e). The peaks corresponding to the bilayer structure found in the specular XRD scans are the 001 and 002. In the patterns, also the third- and fifth-order peaks are present, located at 0.60 and $1.0 \AA^{-1}$, respectively. The peak at $1.25 \AA^{-1}$ is instead attributed to the hexagonal in-plane structure of the smectic B phase with an interplanar distance of $5 \AA$ (Figure 1c) ${ }^{21}$ At low substrate temperature (Figure $2 a$ ), the polymers show a strong preferential orientation along the $z$-axis for the bilayered structure, that is, with the fluorinated chains perpendicular and the polymeric backbone chains parallel to the substrate. The crystalline domains of pPFDA contact the substrate surface along the (001) plane, which is typically referred to as a fiber texture. The peaks showed a broad intensity profile, suggesting small crystallite size. The Bragg peak assigned to the hexagonal in-plane orientation showed a rodlike shape. Bragg rods are generally attributed to $2 \mathrm{D}$ crystals, where little to no correlation is present between the planes parallel to the substrate. ${ }^{32}$

When the substrate temperature is increased to $30{ }^{\circ} \mathrm{C}$ (Figure 2b), diffracted intensity was found distributed along arcs of constant $|\vec{q}|$ (so-called Debye-Scherrer rings). Therefore, a larger spread of crystal planes contacting the substrate surface was present. When intensity is measured along the
Debye-Scherrer rings, the term "mosaicity" is generally adopted to describe the extent of the spread in the crystallite orientation. A higher mosaicity is witnessed as a function of the relative intensity along the Debye-Scherrer ring. An extreme case of mosaicity is powder like texture, that is, when the same intensity is measured along the ring. However, for the pPFDA layers deposited at $30{ }^{\circ} \mathrm{C}$ most of the intensity was still located at the Bragg spots, indicating that the preferential orientation of the lamella with the substrate surface largely persists. Additionally, Bragg peaks appeared sharper both in the GIXD data and in the specular scan (Figure 2f), suggesting an increase in the crystallite size. At $40{ }^{\circ} \mathrm{C}$, two distinct preferential orientations were observed (Figure 2c), with the lamella bilayer oriented either parallel or perpendicular to the substrate surface (schematically depicted in Figure $1 \mathrm{~b}$ ). The appearance of a second crystallite orientation accounts also partially for the sudden intensity decrease observed in specular XRD scans, which are only sensitive to one of the two orientations.

The Bragg rod of the hexagonal in-plane orientation was also partially lost at higher substrate temperature due to a more pronounced randomization of the crystallite orientations. In the literature, an increase in substrate temperature from 30 to $45{ }^{\circ} \mathrm{C}$ showed a similar decrease in crystallinity when measured with specular XRD scans, which was attributed to a more predominant amorphous phase due to the thermal energy provided to the system during synthesis. ${ }^{10}$ In this study, the deeper in-plane structure investigation, instead, showed that increasing the temperature up to $40{ }^{\circ} \mathrm{C}$ allowed the perfluorinated chains to orient parallel to the substrate and to organize the lamella structure with the backbone chains perpendicular to the substrate plane. Increasing the substrate temperature above $40{ }^{\circ} \mathrm{C}$ had a detrimental effect on the preferential orientation of the PFDA crystallites. As reported in Figure $2 \mathrm{~d}$, a powder like spectrum of pPFDA was observed for substrate temperatures of $50{ }^{\circ} \mathrm{C}$. The further increase in thermal energy impedes a regular packing of the pPFDA chains with a distinct contact plane with the substrate surface. The crystallites were then found to orient randomly in the polymer 

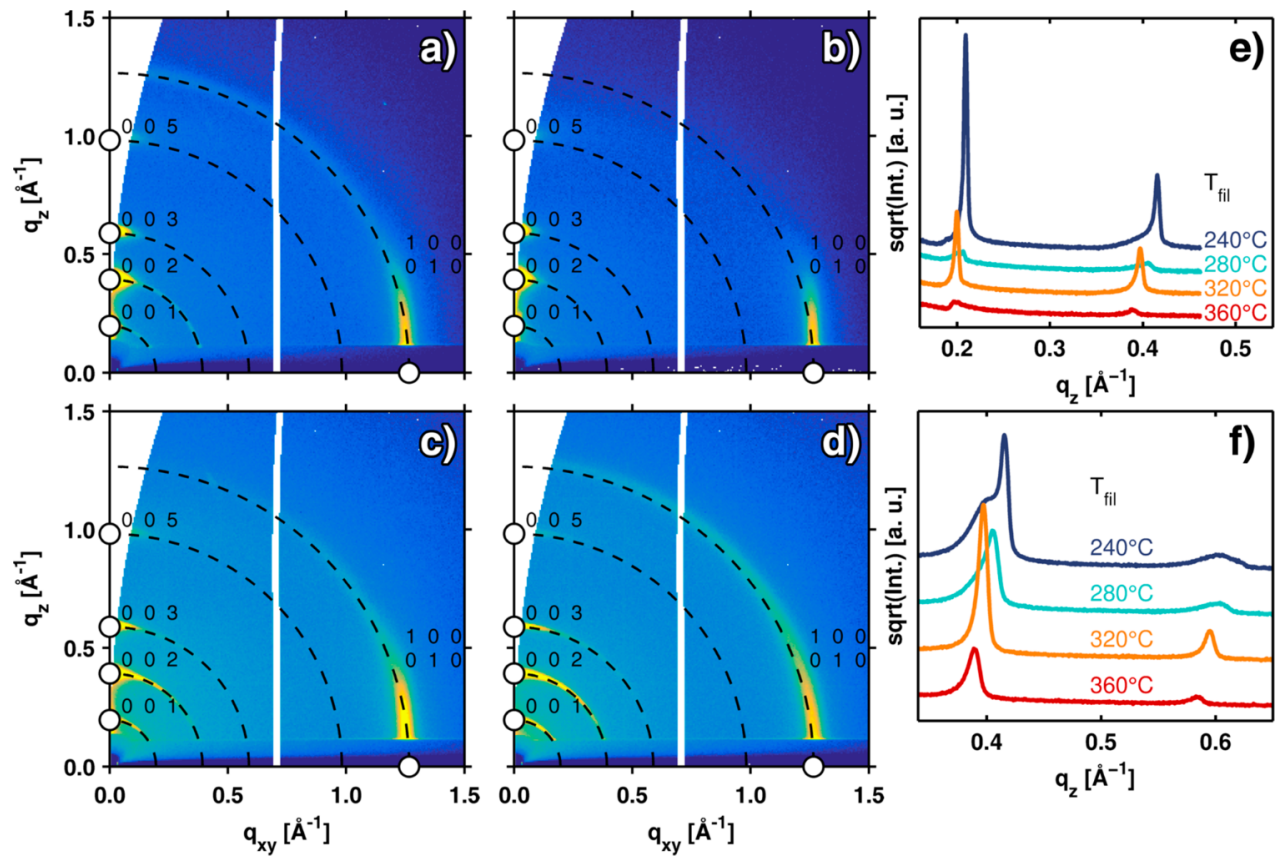

Figure 4. GIXD data for thin pPFDA films deposited by iCVD at a substrate temperature of $30^{\circ} \mathrm{C}$ and at filament temperatures of (a) 240, (b) 280, (c) 320, and (d) $360^{\circ} \mathrm{C}$. (e) Specular XRD patterns of the samples, with a detailed scan of second- and third-order reflections being depicted in (f). Data have been shifted vertically for the sake of clarity.

bulk. Overall, these effects increased the mosaicity of the polymer texture. Finally, a general decrease in intensity along the Debye-Scherrer rings was found at $60{ }^{\circ} \mathrm{C}$, together with an increase in intensity of the background (Figure 2e). The more pronounced halo is generally attributed to an amorphous material, meaning that the higher monomer mobility does not allow for regular growth of the crystallites during deposition. In a previous study, the postdeposition thermal stability of pPFDA was evaluated, evidencing the melting point of the lamella at $73 \pm 2{ }^{\circ} \mathrm{C} .^{30}$ Most likely, the higher mobility of the growing chains during the deposition along with the additional thermal energy provided to the monomers adsorbed on the surface induces a loss of order, yielding a mostly amorphous polymer.

To probe the surface morphology, atomic force microscopy images were taken for the different pPFDA films, depicted in Figure $3 \mathrm{a}-\mathrm{e}$. Increasing the temperature from $20{ }^{\circ} \mathrm{C}$ (Figure 3a) to $40{ }^{\circ} \mathrm{C}$ (Figure 3c), the polymer surface showed the appearance of defined features, indicating an island growth of the polymer. In the literature, the appearance of defined structured features was reported for pPFDA layers deposited from solution when the solvent was quickly evaporated. ${ }^{27}$ At low temperatures, smoother layers were obtained $\left(\sigma_{\mathrm{RMS}}=4\right.$ $\mathrm{nm}$ ), in contrast with literature values for similar systems. ${ }^{10}$ Furthermore, complete adhesion of the layer to the substrate was witnessed, and no large-scale dewetting of the polymer layer from the Si substrate was observed. However, it should be noted that smaller vacancies are evident from the AFM images (e.g., the dark spots in Figure $3 \mathrm{~b}, \mathrm{c}$ ). In a previous investigation, ${ }^{10}$ the phenomenon of dewetting was ascribed to the low surface energy of the fluorinated polymer together with the possible inclusion of unreacted monomer and short oligomers. The more volatile species would then desorb from the polymer bulk, causing the formation of holes in the polymer. The absence of dewetting together with the smoother surface for the layer observed in the present study might be related to differences in the experimental conditions (e.g., a working pressure of $200 \mathrm{mT}$ orr compared to one of $800 \mathrm{mT}$ orr in the present study) as well as differences in reactor geometry influencing the transport of monomer and radical initiator molecules. In the conditions explored, the amount of monomer adsorbed on the surface is close to saturation (with a $P_{\mathrm{M}} / P_{\text {sat }}$ value of 0.9 , see the Supporting Information) for very low substrate temperature. The high amount of PFDA monomer adsorbed on the substrate surface might induce a more homogeneous growth, limiting the formation of short oligomers and compensating for the low surface energy of pPFDA. In Figure $3 f$, the surface roughness $\left(\sigma_{\mathrm{RMS}}\right)$ and the autocorrelation length $\tau$ are reported as a function of the substrate temperature. The autocorrelation length can serve as a measure for mean surface structure size (i.e., the radius). Both parameters were obtained from an analysis of the radial power spectral density function (rPSDF) of the data. The rPSDF is based on Fourier decomposition of the image into specific spatial frequencies, deconvoluting the surface roughness as a function of unit length. ${ }^{33}$ Increasing the temperature from 20 to $30{ }^{\circ} \mathrm{C}$ caused the growth of more pronounced islands, leading to a dramatic increase of the surface roughness to $130 \mathrm{~nm}$. Static water contact angle measurements (see Figure S4) showed a superhydrophobic behavior $\left(>150^{\circ}\right)$ for these layers, a consequence of the high surface roughness. ${ }^{34,35}$ When compared to pPFDA deposited from solutions, the surface roughness value is 1 order of magnitude lower than the ones obtained with iCVD, ${ }^{27}$ inferring that the presence of solvents suppresses the structuration of the pPFDA polymer. Interestingly, the lateral dimension of the islands was only slightly increased, suggesting a preferential out-of-plane anisotropic growth of the polymer. The islands have an elongated form, randomly oriented on the polymer surface. This is in agreement with the GIXD images that indicate an increase of the mosaicity of the polymer with temperature while still maintaining a preferential orientation of the bilayers 

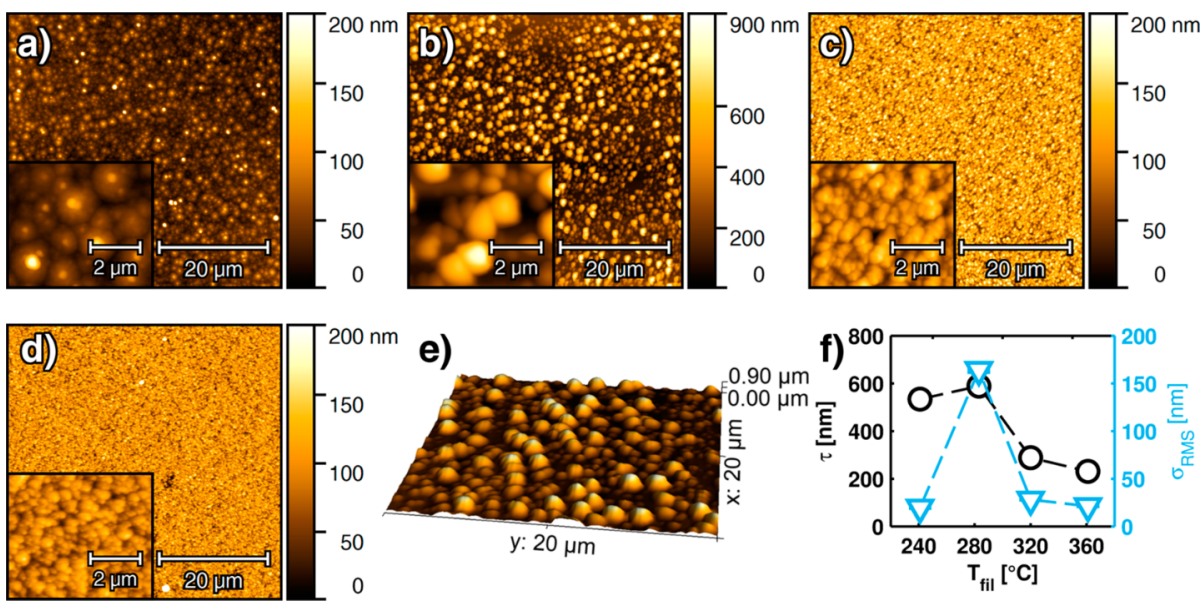

Figure 5. Topographical AFM images for iCVD pPFDA thin films deposited with filament temperatures of (a) 240, (b) 280, (c) 320 , and (d) 360 ${ }^{\circ} \mathrm{C}$. Insets: AFM images with higher resolution. (e) 3D representation of the AFM image reported in (b), showing the isolated columnar growth of pPFDA. (f) Surface roughness $\sigma_{\mathrm{RMS}}$ and the autocorrelation length $\tau$ as determined from analysis of the radial power spectral density function (rPSDF) of the data.

(fiber texture). At $40{ }^{\circ} \mathrm{C}$, the islands were found to grow, reaching a lateral diameter of about $2 \mu \mathrm{m}$ and completely covering the surface, while the surface roughness was found to be fairly constant. The lateral growth of the grains on the polymer surface can be attributed to the presence of the two preferential orientations of the bilayer (with respect to the substrate surface), as evidenced by the GIXD data. The orientations of the fluorinated chains, both perpendicular and parallel to the substrate surface, would induce a more isotropic and disordered growth of the crystals, accounting for the increased lateral dimension and high roughness. In this condition, superhydrophobicity and nonadhesion (slippery behavior) were achieved, probably due to the transition from a Wenzel to a Cassie-Baxter surface (see Figure S4). ${ }^{36}$ Furthermore, polarized optical microscopy images performed on these layers highlighted the highly crystalline nature of the islands (Figure S7). A sudden change in the surface topography was observed at temperatures between 50 and 60 ${ }^{\circ} \mathrm{C}$. The surface roughness and the features' lateral diameter dramatically dropped to values of 25 and $600 \mathrm{~nm}$, respectively. This is accounted for by the powder like growth of smaller pPFDA crystals, which are able then to cover the surface and arrange so as to reduce the surface roughness.

In summary, the substrate temperature was found to greatly affect the degree of crystallinity, the crystal orientation, and the topography of the pPFDA layers. At substrate temperatures from 20 to $40{ }^{\circ} \mathrm{C}$, the pPFDA layers were found to develop from a fiber texture to a mixture of two distinct bilayer orientations (parallel and perpendicular to the substrate), which increases the surface roughness by more than 1 order of magnitude. Raising the substrate temperature further induces a powder like texture growth and smoother surfaces, instead, eventually leading to a loss of overall crystallinity. Tuning of the degree of crystallinity and surface morphology can have high technological interest when a specific surface roughness is desired.

Filament Temperature Effect. In order to investigate the effect of the filament temperature on the structure and topography of pPFDA layer, polymers were deposited with filament temperatures in the range $240-370{ }^{\circ} \mathrm{C}$ and with substrate temperatures of 30 and $50{ }^{\circ} \mathrm{C}$. The GIXD and specular XRD measurements for the layers deposited at $30{ }^{\circ} \mathrm{C}$ are presented in Figure 4.

The filament temperature is expected to influence solely the decomposition pathways of the labile radical initiator, while keeping the structure of the monomer intact. At temperatures below $270{ }^{\circ} \mathrm{C}$, the initiator TBPO is known to be cleaved at the peroxide bond, forming two tert-butoxy radicals. When the temperature is increased above $270{ }^{\circ} \mathrm{C}$, a second cleavage takes place through the $\beta$-scission of the tert-butoxy radicals, leading to the formation of smaller methyl radicals. ${ }^{37}$ Because of the different dimension of the formed radicals $(0.6$ and $0.3 \mathrm{~nm}$ in kinetic diameter for the tert-butoxy and methyl radicals, respectively ${ }^{a}$ ), the filament temperature was shown to affect the crystal orientation as well as the topography of the layer. In a previous contribution, ${ }^{10}$ we reported on the effect of the filament temperature on the pPFDA structure, analyzing polymers obtained with filament temperatures in the range 240-300 ${ }^{\circ} \mathrm{C}$. At low filament temperatures, the predominant crystal orientation was found to be with the lamella oriented perpendicular to the substrate (as shown in Figure 1c, bottom). At high filament temperature, instead, the smaller radicals were found to more easily access also monomers organized perpendicularly on the substrate surface, and the lamella is then aligned parallel to the substrate. AFM images also evidenced an increase in surface roughness as a function of the filament temperature.

In the present study, a deviating behavior was observed. In Figure $4 a-d$, GIXD data of pPFDA films prepared at different filament temperatures are depicted. All the samples exhibit a preferential texture, with the lamella being oriented parallel to the substrate. However, with increased filament temperature also a weak powder like intensity distribution is noted, indicating the formation of more randomly oriented domains. This is also evident when comparing the XRD pattern collected with different detector settings. While differences in intensity appear much larger in a truly specular scan (Figure $4 \mathrm{e})$, they become less pronounced when taking also some offspecular intensity ( spread $\left.\Delta \omega= \pm 1.65^{\circ}\right)$ into account (Figure $4 \mathrm{f})$. Interestingly, the diffraction pattern of the pPFDA layer deposited at a filament temperature of $240{ }^{\circ} \mathrm{C}$ is exhibiting a rather peculiar Bragg peak pattern (Figure 4e). Together with the peaks at 0.20 and $0.40 \AA^{-1}$, an additional peak is visible at 
$0.42 \AA^{-1}$, corresponding to a planar spacing of $15 \AA$. This spacing is smaller than the second order of the bilayer (15.7 $\AA$ ), suggesting that a different organization of the fluorinated chains may occur when only tert-butoxy radicals are initiating the polymer growth. The radicals might induce a distortion in the bilayer structure, which would also account for the asymmetric peak at $0.20 \AA^{-1}$. In the literature, the bilayer structure is also reported in an alternating configuration, in which the fluorinated chains of two different polymeric backbones are not facing each other, but instead interdigitating from the two different chains. ${ }^{38}$ In this configuration, interpenetration of the perfluorinated chains is also possible, reducing the overall spacing of the bilayer and possibly accounting for the additional peaks. While such a behavior can also be noted in previously published data, ${ }^{10}$ it has not been explicitly addressed, and further investigations will be needed to confirm this hypothesis. The peak disappeared when increasing the filament temperature to $280{ }^{\circ} \mathrm{C}$, and the peak position was found to shift toward the lamella Bragg peak at $0.40 \AA^{-1}$. At this temperature, both tert-butoxy and methyl radicals should be present. This competition in the chain initiation would lead to a higher degree of disorder of the polymer growth, thus accounting for the lower crystallinity and the larger mosaicity. A further increase in filament temperature was found to recover the degree of crystallinity of the polymeric structure together with a higher mosaicity (Figure 4c). Finally, increasing the filament temperature up to $360{ }^{\circ} \mathrm{C}$ led to a stronger mosaicity in the polymer crystal structure, as inferred from the increased intensity along the rings in the GIXD patterns, and the consequential decrease of intensity in the XRD specular scan (Figure 4e).

The topographical AFM images of the pPFDA polymer as a function of the filament temperature are reported in Figure 5.

At low filament temperature $\left(240{ }^{\circ} \mathrm{C}\right.$, Figure $\left.5 \mathrm{a}\right)$, the layers are characterized by a low surface roughness $(19.7 \pm 0.2 \mathrm{~nm})$ but a high lateral radius of the surface features $(\tau=530 \pm 5$ $\mathrm{nm}$ ). The structures observed with AFM pointed out an island growth with subsequent coalescence of the growing islands. The low surface roughness is likely linked to the prevalence of the tert-butoxy radicals at this filament temperature; although bulky, a much smaller number of these radicals is present during deposition and, in turn, also included in the final polymer. When methyl radicals are expected to form (filament temperature $280^{\circ} \mathrm{C}$, Figure $5 \mathrm{~b}$ ), a more disordered growth of the polymer was observed, as already aforementioned. Together with a decrease in the overall crystallinity (Figure 4b), the island growth becomes asymmetric, with features resembling a columnar type growth. A more comprehensive representation of the polymer structure is also reported in Figure 5e. The surface roughness is greatly affected by the columnar growth, reaching values of $160 \pm 2 \mathrm{~nm}$ and resulting in superhydrophobic behavior (Figure S4). A similar phenomenon was reported by Vilaró et al. ${ }^{5}$ In their study, PFDA was copolymerized by iCVD with EGDMA, and the film showed a wormlike shape growth, with protuberances smaller than $100 \mathrm{~nm}$ in radius arising out of a uniform layer. This effect was attributed to the combination of a microstructuring of the substrate (obtained via plasma etching prior to deposition) together with the presence of the comonomer EGDMA. The latter creates the conditions for a uniform layer from which the nanofeatures can grow. In a similar fashion, the columnar growth observed in the present study could be attributed to the presence of two competing growth modes which create the conditions for an anisotropic polymerization and micro/nano structures. The growth of a more uniform layer when the methyl radical initiated the polymerization is confirmed by the AFM images of the pPFDA layers deposited at higher filament temperatures $\left(320\right.$ and $360{ }^{\circ} \mathrm{C}$, Figures $5 \mathrm{c}$ and $5 \mathrm{~d}$, respectively). When the dominant radical formation is the $\beta$-scission, smaller, more uniform structures are observed on the surface. The surface roughness is dramatically reduced $(28.2 \pm 0.2 \mathrm{~nm}$, Figure $5 \mathrm{f})$ together with the lateral diameter of the surface features (Figure 5f). A further increase of the filament temperature enhanced this phenomenon, creating smoother surfaces and smaller surface features.

In summary, it was shown that the filament temperature can be used to tune the surface structure and the crystallographic orientation (texture). At low filament temperatures the preferential orientation is with the bilayer lamella perpendicular to the surface, inducing an island growth of big isolated structures. Increasing the filament temperature first leads to a more anisotropic growth of the polymer layer, with columnar features arising from the more uniform surface, reducing the overall crystallinity of the layer. Increasing the temperature further would make the methyl radical the dominant polymerization initiator. In this regime, smaller features are formed, which lead to smoother surfaces and more randomly oriented crystals.

Finally, the effects of both substrate and filament temperature were investigated. In Figure 6, the main results of the
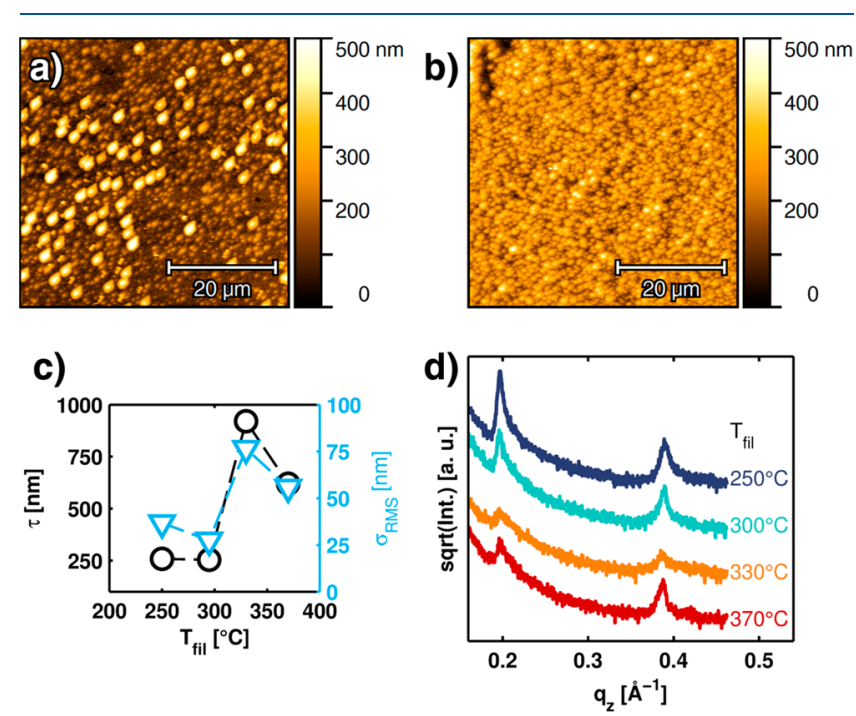

Figure 6. Topographical AFM images of the pPFDA layers deposited at substrate temperature of $50{ }^{\circ} \mathrm{C}$ and filament temperatures of (a) 330 and (b) $370{ }^{\circ} \mathrm{C}$. (c) Surface roughness $\sigma_{\mathrm{RMS}}$ and the autocorrelation length $\tau$ for all the samples. (d) Specular XRD data as a function of filament temperature. The plots are stacked along the $y$-axis for the sake of clarity.

morphological and topographical analysis are reported for pPFDA thin films deposited at $50{ }^{\circ} \mathrm{C}$ in substrate temperature, changing the filament temperature from 250 to $370^{\circ} \mathrm{C}$, and compared with the results of Figure 5 at $30{ }^{\circ} \mathrm{C}$.

As previously mentioned, the layers deposited at higher substrate temperatures are generally smoother and with a higher mosaicity (see Figures 2 and 3). However, as also observed for the layer deposited at $30{ }^{\circ} \mathrm{C}$, when increasing the filament temperature, a competitive growth initiated by the tert-butoxy and methyl radicals is observed. However, 
increasing the substrate temperature and, in turn, the mobility of species on the surface seemed to delay the columnar growth observed for layers deposited with a filament temperature of $280{ }^{\circ} \mathrm{C}$ (Figure $5 \mathrm{~b}$ ). The layers deposited at higher substrate temperatures showed a retention of low surface roughness and lateral feature radius when using filament temperatures up to $300{ }^{\circ} \mathrm{C}$ (Figure 6c), with values in the ranges of $27-37$ and 250-260 nm, respectively. This result pointed out the possible suppression of the chain initiation attributed to the methyl radicals at higher substrate temperature. Under these conditions, a higher mobility and lower surface adsorption could affect the degree of polymerization initiated by this specific radical type. The formation of columnar isolated structures, a possible indication of the competitive growth between the two types of radicals, is observed at $330{ }^{\circ} \mathrm{C}$, as reported in Figure 6a,c. The roughness was found to increase to values of $75 \mathrm{~nm}$, together with a lateral radius of surface features in the order of $900 \mathrm{~nm}$. At this filament temperature, the amount of methyl radicals that are generated is high enough to compensate for the higher mobility and lower surface adsorption, triggering the competitive growth. The water contact angle showed superhydrophobic behavior for these layers, as a consequence of the high surface roughness (Figure S4). Increasing the filament temperature even further reduces this phenomenon, and smoother layers with more homogeneously distributed surface structures were observed (Figure 6b). The crystallinity followed the aforementioned trend as a function of the morphology and competitive growth mechanisms. The lower degree of crystallinity was found for the layer showing the highest surface roughness, deposited at $330{ }^{\circ} \mathrm{C}$. At both higher and lower filament temperatures, the degree of crystallinity is comparable (Figure 6d). In the XRD scan, only peaks attributed to the bilayer lamella were found. The additional peak at $0.42 \AA^{-1}$ is not present when increasing the temperature of the substrate to $50{ }^{\circ} \mathrm{C}$. The higher mobility of the monomer on the surface during the growth was probably sufficient to avoid distorted or alternative crystal organizations.

\section{CONCLUSIONS}

In the present work, more insights into the growth modes and crystal organization of pPFDA layers deposited by iCVD were presented. A clear dependence of the morphology and crystal structure from the process parameters explored was observed. The possibility to finely tune these parameters can have a high impact on applications where specific surface properties (such as roughness and wettability) are sought. Moreover, additional insights into the polymer growth represent useful tools for the fundamental understanding of iCVD processes.

Low substrate temperatures were found to lead to smooth, crystalline layers, with a preferential orientation in the fiber structure. As the substrate temperature is increased, a process window where both orientation and roughness of the pPFDA can be tuned was identified. At $40{ }^{\circ} \mathrm{C}$, two preferential orientations were found, with the bilayer both parallel and perpendicular to the substrate surface. The presence of both orientations leads to a peak in the surface roughness, tunable between 4 and $130 \mathrm{~nm}$ with substrate temperature. A further increase of the substrate temperature leads to a loss of crystallinity and smoothening of the polymer surface.

A variation of the filament temperature could also be used to tune the morphology and crystal structure. At low filament temperature, the dominance of the tert-butoxy radicals initiating the polymerization caused the formation of a fiber- like structure with a possible distortion of the lattice due to the steric hindrance of the initiator. Increasing the filament temperature additionally leads to the formation of methyl radicals. The competition between the two types of initiator radical in growing the polymer chains can be related to the formation of columnar structures, increasing the surface roughness up to $150 \mathrm{~nm}$. When the methyl radical initiator is instead dominant, loss of preferred growth was found, with a drop in the roughness and increased mosaicity of the polymer crystal structure.

In the experimental conditions explored in this study, it was possible to steer the film properties and tailor the material characteristics, which might be relevant for specific applications. The possibility to range from rough, highly crystalline layers (advantageous in fields like biomaterials) to smooth amorphous layers (adopted for instance in optical applications) can be of a high technological interest. Differences to what has previously been reported in the literature hint at other influencing factors (such as pressure and reactor geometry), which affect film growth in iCVD processes. For this reason, more investigations would be necessary in order to identify a general process window and, in turn, the possibility to finely predict the film properties.

\section{ASSOCIATED CONTENT}

\section{Supporting Information}

The Supporting Information is available free of charge on the ACS Publications website at DOI: 10.1021/acs.macromol.8b00904.

$P_{\mathrm{M}} / P_{\text {sat }}$ values for the process conditions; pPFDA FTIR spectrum; pPFDA XPS C 1s high-resolution spectrum and peak assignment; detailed specular X-ray diffraction pattern of thin pPFDA films deposited by iCVD at different substrate temperatures; static water contact angle measurements for all the conditions explored; GIXD data for thin pPFDA films deposited by iCVD at different filament temperatures with a substrate temperature of $50{ }^{\circ} \mathrm{C}$; topographical AFM images for iCVD pPFDA thin films deposited with filament temperatures of 250 and $295{ }^{\circ} \mathrm{C}$ (substrate temperature set at $50{ }^{\circ} \mathrm{C}$ ); powder scans obtained from the GIXD maps by integrating the intensity over the Debye-Scherrer rings (PDF)

\section{AUTHOR INFORMATION}

\section{Corresponding Author}

*E-mail anna.coclite@tugraz.at (A.M.C.).

ORCID

Alberto Perrotta: 0000-0002-7007-6153

Andrew O. F. Jones: 0000-0001-9624-6643

Anna Maria Coclite: 0000-0001-5562-9744

\section{Notes}

The authors declare no competing financial interest.

\section{ACKNOWLEDGMENTS}

The authors thank Elettra Sincrotrone Trieste for the allocation of synchrotron radiation beamtime and L. Barba and $\mathrm{M}$. Polentarutti for providing assistance with the beamline XRD1. The financial support of the FWF-ProCVD project ( $P$ 26993-N19) is also gratefully acknowledged. 


\section{ADDITIONAL NOTE}

${ }^{a}$ The molecular geometry of the molecules was optimized with the Chem3D software. The molecular dimensions were obtained with the bounding box utility of the Mol2Mol software.

\section{REFERENCES}

(1) Bhushan, B.; Jung, Y. C. Natural and Biomimetic Artificial Surfaces for Superhydrophobicity, Self-Cleaning, Low Adhesion, and Drag Reduction. Prog. Mater. Sci. 2011, 56 (1), 1-108.

(2) Oliveira, S. M.; Alves, N. M.; Mano, J. F. Cell Interactions with Superhydrophilic and Superhydrophobic Surfaces. J. Adhes. Sci. Technol. 2014, 28 (8-9), 843-863.

(3) Cohen, N.; Dotan, A.; Dodiuk, H.; Kenig, S. Superhydrophobic Coatings and Their Durability. Mater. Manuf. Processes 2016, 31 (9), $1143-1155$.

(4) Qing, Y.; Yang, C.; Hu, C.; Zheng, Y.; Liu, C. A Facile Method to Prepare Superhydrophobic Fluorinated Polysiloxane/ZnO Nanocomposite Coatings with Corrosion Resistance. Appl. Surf. Sci. 2015, $326,48-54$

(5) Vilaró, I.; Yagüe, J. L.; Borrós, S. Superhydrophobic Copper Surfaces with Anticorrosion Properties Fabricated by Solventless CVD Methods. ACS Appl. Mater. Interfaces 2017, 9 (1), 1057-1065.

(6) Di Mundo, R.; Gristina, R.; Sardella, E.; Intranuovo, F.; Nardulli, M.; Milella, A.; Palumbo, F.; d'Agostino, R.; Favia, P. Micro-/ Nanoscale Structuring of Cell-Culture Substrates with Fluorocarbon Plasmas. Plasma Processes Polym. 2010, 7 (3-4), 212-223.

(7) Liu, S.; Liu, X.; Latthe, S. S.; Gao, L.; An, S.; Yoon, S. S.; Liu, B.; Xing, R. Self-Cleaning Transparent Superhydrophobic Coatings through Simple Sol-gel Processing of Fluoroalkylsilane. Appl. Surf. Sci. 2015, 351, 897-903.

(8) Servi, A. T.; Guillen-Burrieza, E.; Warsinger, D. M.; Livernois, W.; Notarangelo, K.; Kharraz, J.; Lienhard V, J. H.; Arafat, H. A.; Gleason, K. K. The Effects of ICVD Film Thickness and Conformality on the Permeability and Wetting of MD Membranes. J. Membr. Sci. 2017, 523, 470-479.

(9) Coclite, A. M.; Shi, Y.; Gleason, K. K. Grafted Crystalline PolyPerfluoroacrylate Structures for Superhydrophobic and Oleophobic Functional Coatings. Adv. Mater. 2012, 24 (33), 4534-4539.

(10) Coclite, A. M.; Shi, Y.; Gleason, K. K. Controlling the Degree of Crystallinity and Preferred Crystallographic Orientation in PolyPerfluorodecylacrylate Thin Films by Initiated Chemical Vapor Deposition. Adv. Funct. Mater. 2012, 22 (10), 2167-2176.

(11) Milella, A.; Di Mundo, R.; Palumbo, F.; Favia, P.; Fracassi, F.; d'Agostino, R. Plasma Nanostructuring of Polymers: Different Routes to Superhydrophobicity. Plasma Processes Polym. 2009, 6 (6-7), 460466.

(12) Milella, A.; Palumbo, F.; Favia, P.; Cicala, G.; d'Agostino, R. Continuous and Modulated Deposition of Fluorocarbon Films from C-C4F8 Plasmas. Plasma Processes Polym. 2004, 1 (2), 164-170.

(13) Pospiech, D.; Jehnichen, D. Self-Organizing Semifluorinated Methacrylate Copolymers. In Handbook of Fluoropolymer Science and Technology; John Wiley \& Sons, Inc.: Hoboken, NJ, 2014; pp 235290.

(14) Yagüe, J. L.; Gleason, K. K. Vapor Deposition of Fluoropolymer Surfaces. In Handbook of Fluoropolymer Science and Technology; John Wiley \& Sons, Inc.: Hoboken, NJ, 2014; pp 131-148.

(15) Ozaydin-Ince, G.; Coclite, A. M.; Gleason, K. K. CVD of Polymeric Thin Films: Applications in Sensors, Biotechnology, Microelectronics/Organic Electronics, Microfluidics, MEMS, Composites and Membranes. Rep. Prog. Phys. 2012, 75 (1), 016501.

(16) Alf, M. E.; Asatekin, A.; Barr, M. C.; Baxamusa, S. H.; Chelawat, H.; Ozaydin-Ince, G.; Petruczok, C. D.; Sreenivasan, R.; Tenhaeff, W. E.; Trujillo, N. J.; Vaddiraju, S.; Xu, J.; Gleason, K. K. Chemical Vapor Deposition of Conformal, Functional, and Responsive Polymer Films. Adv. Mater. 2010, 22 (18), 1993-2027.

(17) Gupta, M.; Gleason, K. K. Initiated Chemical Vapor Deposition of PFDA Thin Films. Langmuir 2006, 22 (10), 10047-10052.
(18) Ma, M.; Mao, Y.; Gupta, M.; Gleason, K. K.; Rutledge, G. C. Superhydrophobic Fabrics Produced by Electrospinning and Chemical Vapor Deposition. Macromolecules 2005, 38 (23), 9742-9748.

(19) Lau, K. K. S.; Gleason, K. K. Initiated Chemical Vapor Deposition (ICVD) of Poly(Alkyl Acrylates): An Experimental Study. Macromolecules 2006, 39 (10), 3688-3694.

(20) Christian, P.; Coclite, A. M. Thermal Studies on Proton Conductive Copolymer Thin Films Based on Perfluoroacrylates Synthesized by Initiated Chemical Vapor Deposition. Thin Solid Films 2017, 635, 3-8.

(21) Ranacher, C.; Resel, R.; Moni, P.; Cermenek, B.; Hacker, V.; Coclite, A. M. Layered Nanostructures in Proton Conductive Polymers Obtained by Initiated Chemical Vapor Deposition. Macromolecules 2015, 48 (17), 6177-6185.

(22) Matin, A.; Khan, Z.; Gleason, K. K.; Khaled, M.; Zaidi, S. M. J.; Khalil, A.; Moni, P.; Yang, R. Surface-Modified Reverse Osmosis Membranes Applying a Copolymer Film to Reduce Adhesion of Bacteria as a Strategy for Biofouling Control. Sep. Purif. Technol. 2014, $124,117-123$.

(23) Martin, T. P.; Lau, K. K. S.; Chan, K.; Mao, Y.; Gupta, M.; Shannan O'Shaughnessy, W.; Gleason, K. K. Initiated Chemical Vapor Deposition (ICVD) of Polymeric Nanocoatings. Surf. Coat. Technol. 2007, 201 (22-23), 9400-9405.

(24) Honda, K.; Morita, M.; Otsuka, H.; Takahara, A. Molecular Aggregation Structure and Surface Properties of Poly(Fluoroalkyl Acrylate) Thin Films. Macromolecules 2005, 38 (13), 5699-5705.

(25) Coclite, A. M.; Lund, P.; Di Mundo, R.; Palumbo, F. Novel Hybrid Fluoro-Carboxylated Copolymers Deposited by Initiated Chemical Vapor Deposition as Protonic Membranes. Polymer 2013, 54 (1), 24-30.

(26) Volkov, V. V.; Platé, N. A.; Takahara, A.; Kajiyama, T.; Amaya, N.; Murata, Y. Aggregation State and Mesophase Structure of CombShaped Polymers with Fluorocarbon Side Groups. Polymer 1992, 33 (6), 1316-1320.

(27) Kim, J.; Efimenko, K.; Genzer, J.; Carbonell, R. G. Surface Properties of Poly[2-(Perfluorooctyl)Ethyl Acrylate] Deposited from Liquid CO 2 High-Pressure Free Meniscus Coating. Macromolecules 2007, 40 (3), 588-597.

(28) Nečas, D.; Klapetek, P. Gwyddion: An Open-Source Software for SPM Data Analysis. Cent. Eur. J. Phys. 2012, 10 (1), 181-188.

(29) Lausi, A.; Polentarutti, M.; Onesti, S.; Plaisier, J. R.; Busetto, E.; Bais, G.; Barba, L.; Cassetta, A.; Campi, G.; Lamba, D.; Pifferi, A.; Mande, S. C.; Sarma, D. D.; Sharma, S. M.; Paolucci, G. Status of the Crystallography Beamlines at Elettra. Eur. Phys. J. Plus 2015, 130 (3), 43.

(30) Christian, P.; Coclite, A. M. Vapor-Phase-Synthesized Fluoroacrylate Polymer Thin Films: Thermal Stability and Structural Properties. Beilstein J. Nanotechnol. 2017, 8 (1), 933-942.

(31) Tazreiter, M.; Christian, P.; Schennach, R.; Grießer, T.; Coclite, A. M. Simple Method for the Quantitative Analysis of Thin Copolymer Films on Substrates by Infrared Spectroscopy Using Direct Calibration. Anal. Methods 2017, 9 (36), 5266-5273.

(32) Meijer, J.-M.; Shabalin, A.; Dronyak, R.; Yefanov, O. M.; Singer, A.; Kurta, R. P.; Lorenz, U.; Gorobstov, O.; Dzhigaev, D.; Gulden, J.; Byelov, D. V.; Zozulya, A. V.; Sprung, M.; Vartanyants, I. A.; Petukhov, A. V. Double Hexagonal Close-Packed Structure Revealed in a Single Colloidal Crystal Grain by Bragg Rod Analysis. J. Appl. Crystallogr. 2014, 47 (4), 1199-1204.

(33) Nenadović, M.; Potočnik, J.; Ristić, M.; Štrbac, S.; Rakočević, Z. Surface Modification of Polyethylene by $\mathrm{Ag}+$ and $\mathrm{Au}+$ Ion Implantation Observed by Phase Imaging Atomic Force Microscopy. Surf. Coat. Technol. 2012, 206 (19-20), 4242-4248.

(34) Favia, P.; Cicala, G.; Milella, A.; Palumbo, F.; Rossini, P.; d'Agostino, R. Deposition of Super-Hydrophobic Fluorocarbon Coatings in Modulated RF Glow Discharges. Surf. Coat. Technol. 2003, 169-170, 609-612.

(35) Hubert, J.; Mertens, J.; Dufour, T.; Vandencasteele, N.; Reniers, F.; Viville, P.; Lazzaroni, R.; Raes, M.; Terryn, H. Synthesis and 
Texturization Processes of (Super)-Hydrophobic Fluorinated Surfaces by Atmospheric Plasma. J. Mater. Res. 2015, 30 (21), 3177-3191.

(36) Di Mundo, R.; Palumbo, F.; D’Agostino, R. Nanotexturing of Polystyrene Surface in Fluorocarbon Plasmas: From Sticky to Slippery Superhydrophobicity. Langmuir 2008, 24 (9), 5044-5051.

(37) Mekarbane, P. G.; Tabner, B. J. Interaction between Radicals Derived from Two Di- $t$-Alkyl Peroxides with Some Monomers and Polymers. Macromolecules 1999, 32 (11), 3620-3625.

(38) De Crevoisier, G.; Fabre, P.; Leibler, L.; Tencé-Girault, S.; Corpart, J. M. Structure of Fluorinated Side-Chain Smectic Copolymers: Role of the Copolymerization Statistics. Macromolecules 2002, 35 (10), 3880-3888. 